\title{
ASSESSMENT AND CLASSIFICATION OF FARM TRACTOR RIMS FOR MECHANICAL TESTING
}

\author{
Marco Bietresato, Francesco Selmo, Tobias Terzer, Fabrizio Mazzetto \\ Libera Universita di Bolzano, Italy \\ marco.bietresato@unibz.it
}

\begin{abstract}
The propulsion system of an agricultural machine is in charge for the delivery of the engine power to the soil and allows a vehicle travelling and performing its duties. Based on the type of this system, agricultural machines can be divided in tracked and wheeled. Although the former have their own specific applications and advantages, the latter are the most widely used. Over the time, different tyre typologies have been developed keeping into account many factors, such as tractor models, sizes and types of field/tillage operations, and wheel rims were changed accordingly. Notwithstanding an attempt of standardization by ISO, the design of these components is still conditioned by manufacturers' previous choices and there are some interchangeability issues. The knowledge of existing differences/dimensions is useful when there is the need for maintenance, substitution or connection of whatever device to a tractor axle. So, with the aim of developing a universal piece of experimental equipment to be used to detect the tractor performances (torque, power) at the wheels, i.e. a hub adapter, a systematic study of the different rim types has been performed. After having collected many data through direct measurements or interviews, all information has been elaborated using many statistical tools, such as scatter plots, frequency charts and clustering algorithms. Finally, four different classification keys have been applied to identify a minimum set of dimensional/functional classes of tractors using similar rims and, hence, capable of using the same hub adapters. The results of this study were then successfully used to design two optimized adapters. Their features (dimensions, number of holes) would let these two adapters connect the test equipment to the axle shafts of up to 1961 tractors of our database, corresponding to $81 \%$ of the farm tractors below $100 \mathrm{~kW}$ on the Italian and European market, thus maximizing the cost-benefit ratio for these components.
\end{abstract}

Keywords: agricultural machines, wheels, tyres, rims, tractor characterization, classification, clustering.

\section{Introduction}

The engine performances of agricultural machinery (in terms of torque and power curves as a function of the engine speed) can be measured in many ways and with different equipment. One of the most common methods for the acquisition of these quantities is connecting a dynamometric brake [16] to the tractor power-take-off (PTO), when the tractor is motionless and the driving wheels are disconnected, and delivering all the engine torque/power to the dyno towards that mechanical connection. According to the second Newton's law of motion, the breaking torque applied by the dyno is instantaneously equal to the engine torque. Unfortunately, the gatherings acquired by this test method are different from the real in-field performances of a tractor, as the losses in the powertrain are completely neglected (the PTO is directly connected to the engine). Actually, there are other test solutions in the market to overcome this problem [7-9], for example: (1) sensorized rims, to be substituted to conventional rims [10-12], able to measure the torque delivered by each wheel of a tractor performing its usual tasks (e.g., when it is working in a field), (2) roller test rig, which applies a braking torque at two or four rollers, each one supporting one driving wheel of the tractor $[13 ; 14]$, (3) four-post dyno test rig, i.e. a test rig composed by four dynos, each one applying a breaking torque to the wheel to which it is connected through a cardan shaft [15]. Although effective, all these pieces of equipment are quite expensive (in particular, the second and the third solution, requiring also a series of adaptations to the buildings that house them). Therefore, we decided to develop, within the "BIO-TRACT-EFFICIENCY" project of the Free University of Bozen/Bolzano, an innovative test rig able to detect the torque and power outputs of a tractor (as well as other parameters) directly at its rear wheels, hence in a measuring point downstream of the powertrain. This test rig will be used to carry out many experiments on the largest possible samples of agricultural vehicles (in terms of power classes, models and age), to pursue the project aim to analyse the operation and efficiency of the whole powertrain (engine + transmission), also when these agricultural machines are powered by alternative fuels. The starting point is a PTO-dyno already owned by the university (a "SIGMA 50 Mobile" PTO-dyno by N. J. Froment \& Co Ltd, Easton-on-the-Hill, East Northamptonshire, England, UK; [1]), useful to apply a breaking torque to the engine, but this device is thought to be connected to the tractor PTO (in terms of operative range and mechanical interfaces) to measure the engine output upstream of the transmission, exploiting the direct connection between the engine and PTO. It is 
therefore necessary to create a mechanical connection between the tractor propulsion elements (wheels) and the PTO-dyno, capable of adapting the wheel rotational speed and torque to the PTO operative range. In this case, the first stage of the connection foresees the use of sprockets (to be mounted on the tractor instead of the wheels) and chains. Therefore, the test rig must be firstly connected to the rear axle shafts of the tractor in test, and the easiest way to do this is using the bolts normally used to fix the rims. The wheel-to-hub fixing system consists of some components that are always present in every type of tractor: a variable number of bolts, support disc and an alignment system (Fig. 1). The dimensions of these components are not always the same, but differ according to the tractor model, type (e.g., universal or special-purpose), construction year and size (i.e., with the maximum power, as will be illustrated later). Notwithstanding an attempt of standardization performed by ISO, dated back to 1995 [16], the design of these components is still conditioned by manufacturers' previous choices and there are some interchangeability issues. For example, according to the data at our disposal (including 2992 models representing the actual Italian tractor pool), $80 \%$ of the tractors built after the year 2000 complies with the cited standard, but, if we consider the whole database, the percentage is sensibly lower: only $43 \%$. This is principally due to the high average age of Italian tractors, which is around 20 years, according to $[17 ; 18]$. Therefore, the actual situation of the tractor pool is extremely diversified and makes extremely difficult the task of a designer to design a hub adapter (or a set of adapters) to connect the test rig to most of tractor models. Therefore, the aim of this study is to perform a systematic study of the different types of rims on the market, thus understanding how many and which types of axle shaft hubs are the most widespread. The results of this study will be then useful for the authors to design the adapter (or a set of adapters) and will also be a valuable tool to guide the designers towards appropriate solutions and limit the component production costs thanks to standardization.

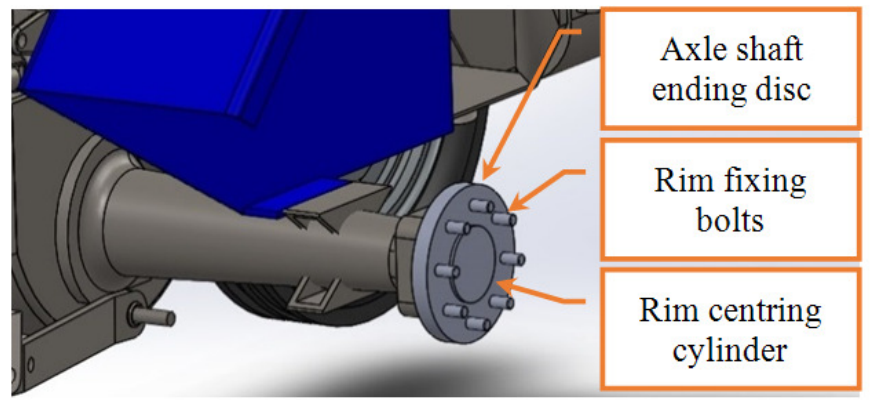

Fig. 1. Example of rim-fastening system on the tractor axle shaft hub

\section{Materials and methods}

In the proposed methodology, before beginning the systematic study of the different rim types, it is necessary to preliminary establish which parameters distinguish and categorize the tractor axle-rim couplings. The ISO 5711 standard [19] concerns both the wheels and the hubs, and describes the basic geometric parameters and reference categories for the rims, which characterize the wheel-to-hub couplings for any type of tractor axle shaft (Fig. 2). With the aim of selecting these parameters, we firstly tried to understand which of the three alignment systems, reported also in the cited standard, is the most used to centre the rim on the tractor axle shaft hub: (1) the rim bore, (2) the rim stud holes for the bolts, with "spherical centring", (3) the rim stud holes for the bolts, with "conical centring". After comparing the data in our database (2992 models of tractors by all the most important manufacturers; Table 1) with the indications given us by the employees of some companies with which we are in contact (in particular: the Farmers' Cooperative of Bolzano, a tractor wrecker in province of Verona and Carraro Agritalia), it was found that the alignment system on the axle shaft hub is preferred by most manufacturers and it is adopted in about $90 \%$ of the models in the market. This evidence allowed us to exclude from the analysis the two centring systems based on the rim holes - hub bolts alignment. Therefore, in particular, the fabrication of a hub adapter (that basically will be mounted on the axle shaft hub instead of the rim) can be done considering only the first three parameters of the ISO 5711 list, plus the number of the equally-spaced holes needed for the bolts. The disc flat bearing diameter (parameter $\mathrm{D}_{4}$ of Fig. 2) is not needed in the design of the adapter, because this one will necessarily have a flat surface in contact with the disc flat bearing, differently from normal rims that 
have a curved shape starting from $\mathrm{D}_{4}$ (to have to so called "offset"), as can be seen in Fig. 2. The centring diameter on the hub $\left(\mathrm{D}_{5}\right)$ and the diameter of the hub flat bearing $\left(\mathrm{D}_{6}\right)$ are two parameters that can be ignored as well, as they refer to redundant characteristic features of the axle ending, hence not directly involved in the hub adapter construction: $D_{5}$ can be derived from $D_{3}$ and $D_{6}$ does not affect the adapter geometry. The parameters of interest for this study are therefore: the number of equally-spaced holes (or, the number of bolts; $\mathrm{N}$ ); the pitch circle diameter (of the wheel-to-hut fixing bolts; $\mathrm{D}_{1}$ ); the stud hole diameter (of the wheel-to-hut fixing bolts; $\mathrm{D}_{2}$ ); the central bore diameter (to be coupled with the rim centring cylinder on the tractor axle shaft; $\mathrm{D}_{3}$ ). The same standard gives also precise indications about the dimensions of the parts to be coupled together. The following Table 2 shows the prescribed dimensions in the case of rims having a "flat attachment type with centring on central bore".

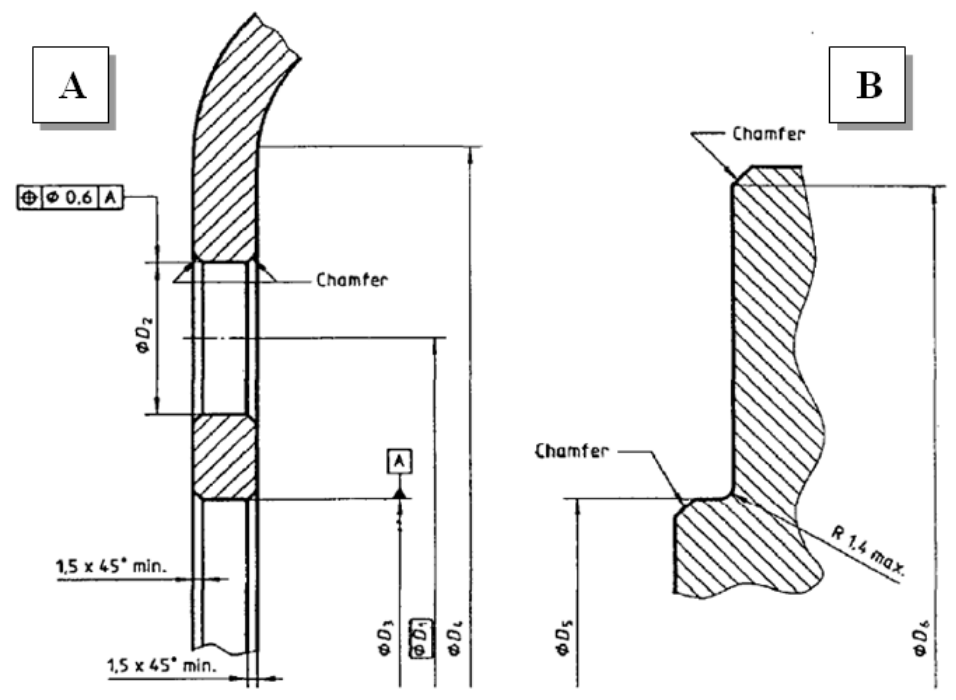

Fig. 2. Wheel-to-hub fixing dimensions from ISO 5711 Standard [19]: A - wheel; B - hub; $\mathrm{D}_{1}$ - pitch circle diameter; $\mathrm{D}_{2}-$ stud hole diameter; $\mathrm{D}_{3}-$ central bore diameter; $\mathrm{D}_{4}$ - disc flat bearing diameter; $\mathrm{D}_{5}$ - centring diameter on the hub; $\mathrm{D}_{6}$ - diameter of the hub flat bearing

Table 1

Tractor manufacturers in the reference database

\begin{tabular}{|c|c|c|c|c|c|}
\hline Carraro & Fiat & John Deere & Mc Cormick & Steyr & Zetor \\
\hline Case & Ford & Kubota & Mercedes & Universal & - \\
\hline Claas & Goldoni & Lamborghini & New Holland & Ursus & - \\
\hline Deutz Fahr & Hürlimann & Landini & Renault & Valpadana & - \\
\hline Fendt & J.C.B. & Massey-Ferg. & Same & Valtra & - \\
\hline
\end{tabular}

Table 2

Prescriptions on dimensions (in mm) for rims having "flat attachment type with centring on central bore" (from ISO 5711 Standard; [19])

\begin{tabular}{|c|c|c|c|c|c|c|c|}
\hline \multirow[b]{2}{*}{$\begin{array}{l}\text { Number of equally } \\
\text { spaced stud holes }\end{array}$} & \multicolumn{4}{|c|}{ Wheel } & \multirow[b]{2}{*}{$\begin{array}{c}\text { Stud } \\
\text { diameter" }\end{array}$} & \multicolumn{2}{|c|}{ Hub } \\
\hline & $\begin{array}{c}D_{1} \\
\text { nom. }\end{array}$ & $\begin{array}{r}D_{2} \\
+1 \\
0\end{array}$ & $\begin{array}{c}D_{3} \\
+0.5 \\
0\end{array}$ & $\begin{array}{l}D_{4} \\
\text { min. }\end{array}$ & & $\begin{array}{c}D_{5} \\
0 \\
-0.2\end{array}$ & $\begin{array}{r}D_{6} \\
0 \\
-5\end{array}$ \\
\hline 4 & 100 & 15 & 61 & 145 & 12 & 60,8 & 140 \\
\hline 5 & 140 & 17 & 96 & 185 & 14 & 95,8 & 180 \\
\hline 6 & 205 & 21 & 161 & 255 & 18 & 160,8 & 250 \\
\hline \multirow{2}{*}{8} & 203.2 & 21 & 152,4 & 257 & 18 & 152,2 & 252 \\
\hline & 275 & 24 & 221 & 325 & 20 & 220,8 & 320 \\
\hline 10 & 335 & 26 & 281 & 390 & 22 & 280.8 & 385 \\
\hline 12 & 425 & 26 & 371 & 470 & 22 & 370,8 & 465 \\
\hline
\end{tabular}


After having collected many data from the tyre manufacturers, a database containing the aboveevidenced geometric dimensions relevant to many tractor models by principal manufacturers was then created. The first performed analysis was a subdivision of the database entries in frequency classes. Then, other statistical techniques have been applied to better divide the whole database, in particular all the techniques belonging to the so-called clustering methods. A cluster is a set of objects/entities/database entries of a homogeneous type, which have similar peculiarities within the cluster (i.e. the clustering keys, decided by the analyst), dissimilar from external objects, i.e. the objects belonging to other clusters [20]. Cluster analysis is used to group objects/entities/database entries and it is a so-called unsupervised classification process, i.e. there are no predefined classes. A good clustering process is expected to have a high similarity level among objects belonging to the same class and a low similarity level with objects outside that class [21]. For this reason, the quality of the procedure depends on the ability to choose and implement parameters and methods that can well describe and measure the similarity between objects. The quality of clustering also depends on its ability to detect patterns hidden within the data [22;23]. In the present case, the similarity criterion for the rims is based on a practical need: simplifying and reducing the number of axle shaft hub adapters to be built, however, allowing them to connect to the test rig as many agricultural vehicles as possible. At the same time, it is therefore necessary to understand if it is possible to create, on a same adapter, some geometric features that can be even very different from each other, but that are functional to widen the usability of that adapter. So, in this case, the clustering process is strictly linked to the design requirements for the adapter. The first example is the following one: if the only difference between some wheel-to-hub couplings is the diameter of the bolts, the adapter can be made suitable to be used with two or three categories of axle shafts by creating holes of the widest requested diameter, without compromising the tightness of the structure with washers and bushings. Therefore, all the coupling variants, having only small differences in the bolt diameter, can be grouped together. Rather, it is more difficult to group the couplings variants that differ in the alignment hub diameter: centring errors in the assembly, due to a wider bore diameter (to include more than one variant), are not admitted in a rotating system since they can cause static and dynamic unbalancing, vibrations and breaks. From a constructive point of view, however, it is possible to provide the adapter with a set of rings that act as spacers that compensate small differences in the bore diameter and help the positioning of the adapter. The proposed solution, geometrically very simple, is also cheap and easy to be manufactured, because it is basically a ring made of standard structural steel (S355JR EN 100252:2004). Finally, the adapter has been drawn using Solidworks 2018 (by Dassault Systèmes, VélizyVillacoublay, France [24]), a parametric 3D-CAD program. The whole approach followed in this study is reported in Fig. 3.

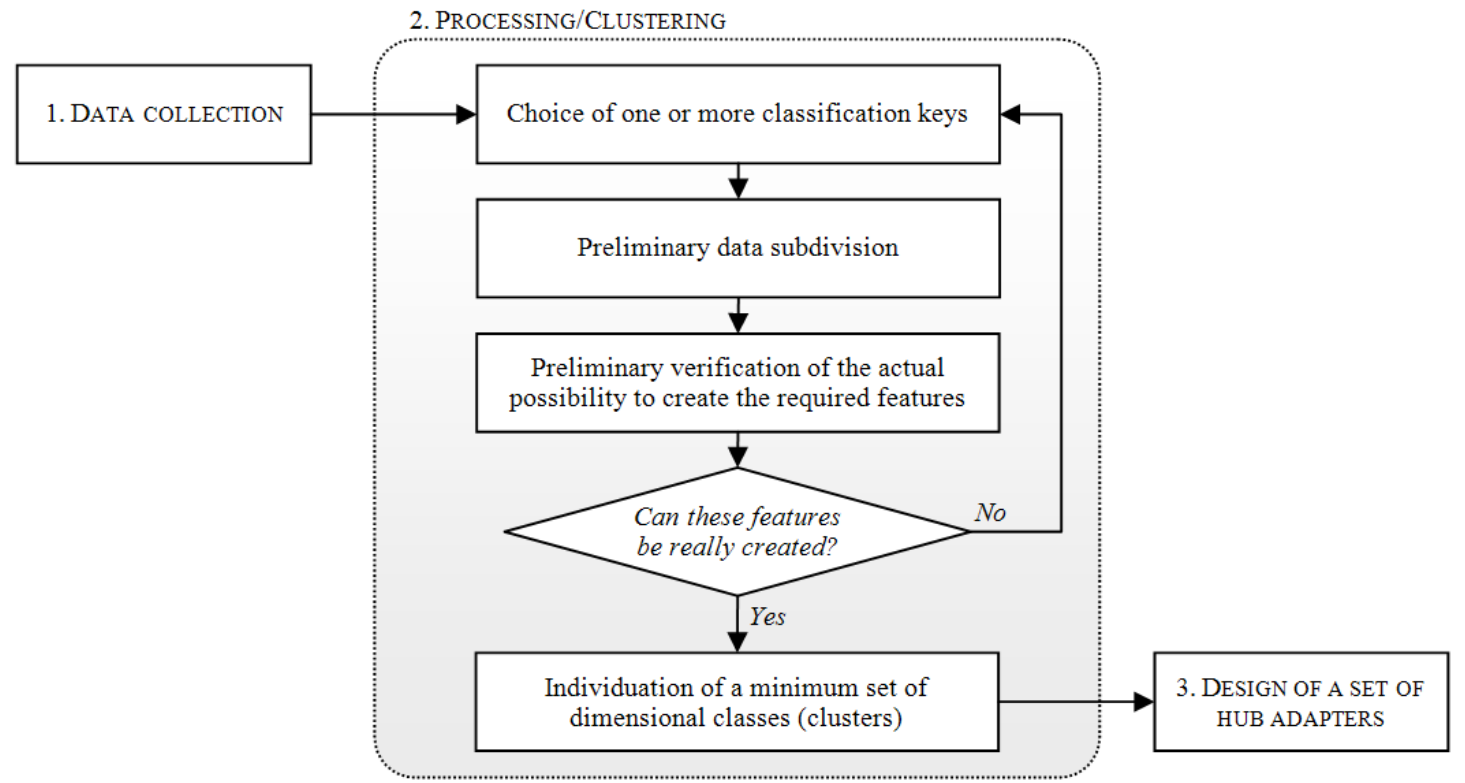

Fig. 3. Three-phase approach followed in the systematic study of the rims to design the adapter 


\section{Results and discussion}

As mentioned above, the dimensional parameters identifying the different rim categories vary essentially in accordance with the maximum power of the tractors on which those rims are installed. For example, as can be seen from the graph of Fig. 4, the pitch circle diameter of the bolts increase with the maximum power of the tractors, witnessed by a thickening of the points along the quadrant bisector (average slope: $2.35 \mathrm{~mm} \cdot \mathrm{kW}^{-1}$ ). This is easy to be understood, if considering the increase of the torque to be delivered by the wheels due to the increase of the maximum power. In the same figure it can also be noticed that the points corresponding to different tractors are not widespread on all the Cartesian plane, even along the bisector. Rather, they are lined up along straight horizontal lines, at ordinate values corresponding to different, well-separated categories of the pitch circle diameter. The range of power values covered by each single class of pitch circle diameter is wide and there are evident overlaps of them along the scale of the power. Consequently, a simple selection of tractors to be tested by the rig under development based on a threshold on the maximum power of testable tractors, does not imply a real limitation of the number of possible pitch circle diameter for the hub adapter, the object of this study. For example, by limiting the maximum power to $150 \mathrm{~kW}$, no pitch circle diameter can be excluded. Limiting the maximum power to $100 \mathrm{~kW}$, only three pitch circle diameters are excluded $(485,425,366 \mathrm{~mm}$ ), but many other measures (precisely 15, from 120 to $335 \mathrm{~mm}$ ) could be still encountered in a tractor. However, considering the Italian and European tractor market $[18 ; 25 ; 26]$, it has been therefore decided to focus on the development of an adapter designed for tractors with a maximum power of $100 \mathrm{~kW}$. Consequently, the database entries (2992 models) have been properly filtered to study only the models with a power below that threshold, thus reducing it to 2417 models (i.e. $81 \%$ of the initial database; hereinafter this will be named "reduced dataset"). As evidenced in the previous section, four main geometrical parameters of the rim were considered for the statistical analyses needed to define the geometry of the adapter (or of the set of adapters): (1) the number of holes, (2) the pitch circle diameter of the rim stud holes for the bolts, (3) the diameter of the central centring bore and (4) the diameter of the rim stud holes for the bolts.

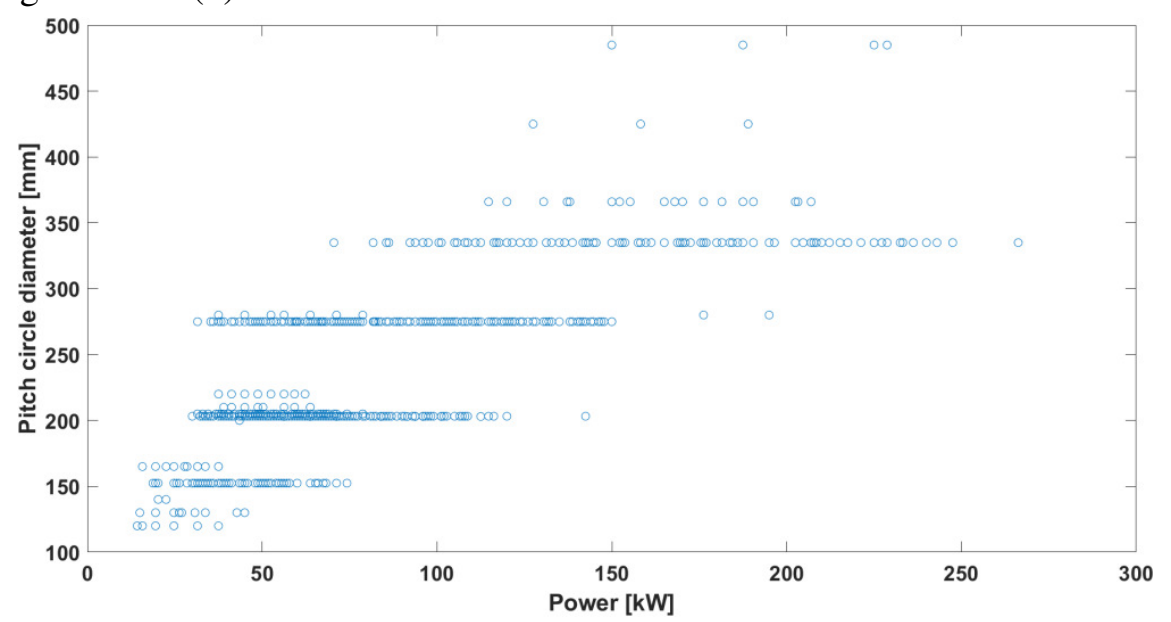

Fig. 4. Pitch circle diameter of bolts as a function of the tractor maximum power

Due to the large variability of bolt hole diameters (up to 14 different measures, probably consequent on independent choices made by the manufacturers on the dimensional tolerances to be used; Fig. 5), the bolt hole diameter was soon replaced by the bolt diameter. According to this new reparametrization, the most frequent measures for the bolt diameter are only 4 over 10 (specifically: 18, 19, 20 and $22 \mathrm{~mm}$ ), as can be seen from Fig. 6.B. By using the same approach for the other three parameters, it is possible to make proper comparisons with the other subdivisions of the reduced dataset Fig.6.B, Fig. 6.C, Fig.6.D). So, it is possible to observe that:

- there are only 2 main categories (over 5) for the variability of the number of bolts used for fastening;

- there are 4 main categories (over 15) for the variability of the pitch circle diameters of the rim stud holes for the bolts;

- there are 7 main categories (over 25) for the variability of the alignment hub diameter. 


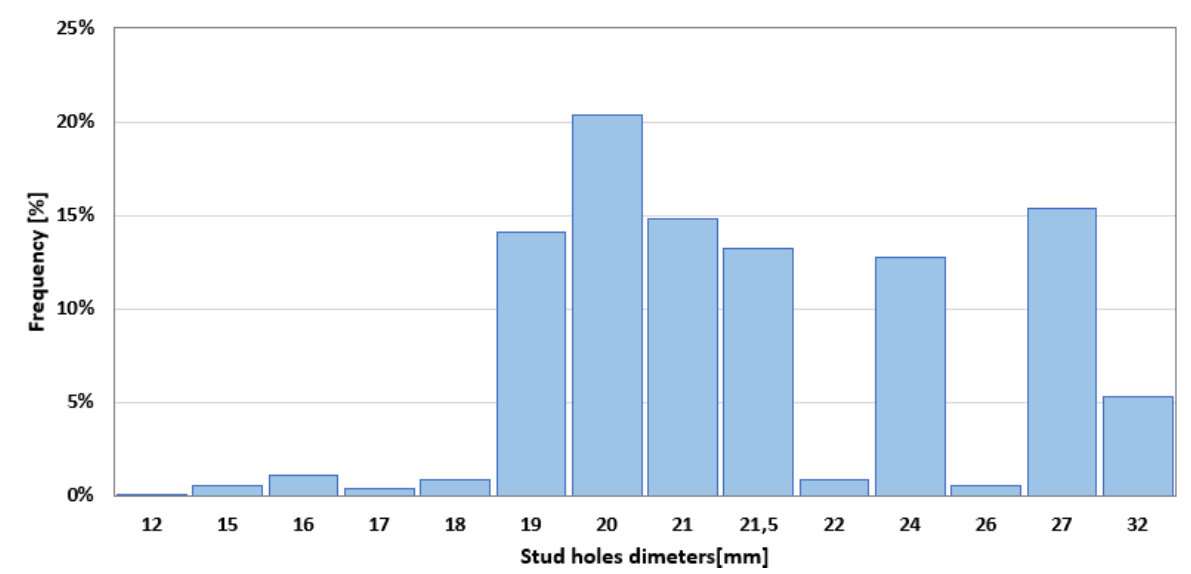

Fig. 5. Variability and occurrence of stud hole diameters (in terms of number of tractor models in the reduced dataset using each category of stud hole diameters)
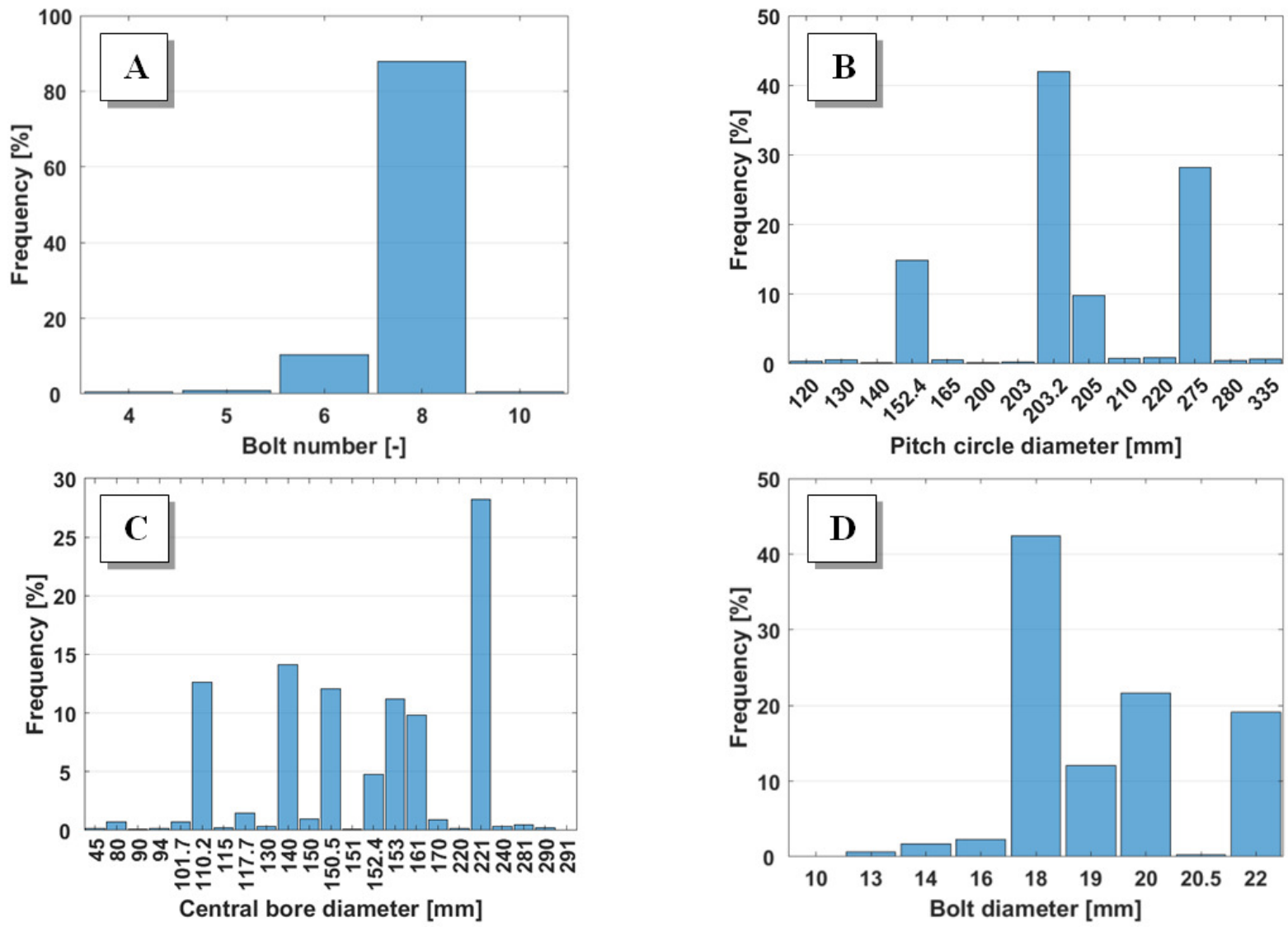

Fig. 6. Variability and occurrence frequency (in terms of number of tractor models in the reduced dataset using that specific value for that parameter) of: $\mathrm{A}$ - number of bolts;

$\mathrm{B}$ - pitch circle diameter of the rim stud holes for the bolts; $\mathrm{C}$ - central centring bore (Hub) diameter; $\mathrm{D}$ - diameter of the bolts

This first analysis allowed to reduce the whole set of combinations of the dimensions for the four parameters $(10 \times 5 \times 15 \times 25=18750)$ to the combinations of parameters that theoretically are most frequently adopted by manufacturers $(4 \times 2 \times 4 \times 7=224)$ and, subsequently, to the combinations of these ones that can be really found on the market. The combinations found in this way are only 38 and, to facilitate their identification, hereinafter each category is defined by a string that indicates explicitly the values of the four fundamental parameters, for example: "N8-P275-H221-B18". The first number concerns the number of bolts on a rim $(N)$, the second is the pitch circle diameter $(P)$, the third is the diameter of the centring hub $(H)$ and the fourth is the diameter of the bolts $(B)$. The multiple variants of coupling systems have been reported on a histogram (Fig. 7), where the height of each column 
indicates the frequency of tractor models with the reported combination of parameters. It is immediately noticeable that some categories have a very low numerousness, whilst the ones with an occurrence greater than $1 \%$ are 10 in total. For example, the category N8-P275-H221-B22 is the most frequently used in tractors under $100 \mathrm{~kW}$, because up to 419 models $(17.3 \%$ of the total) use this combination of parameters. According to this analysis, a set of only 10 adapters would be suitable for a total of 2228 models of tractors, corresponding to $92 \%$ of the reduced dataset.

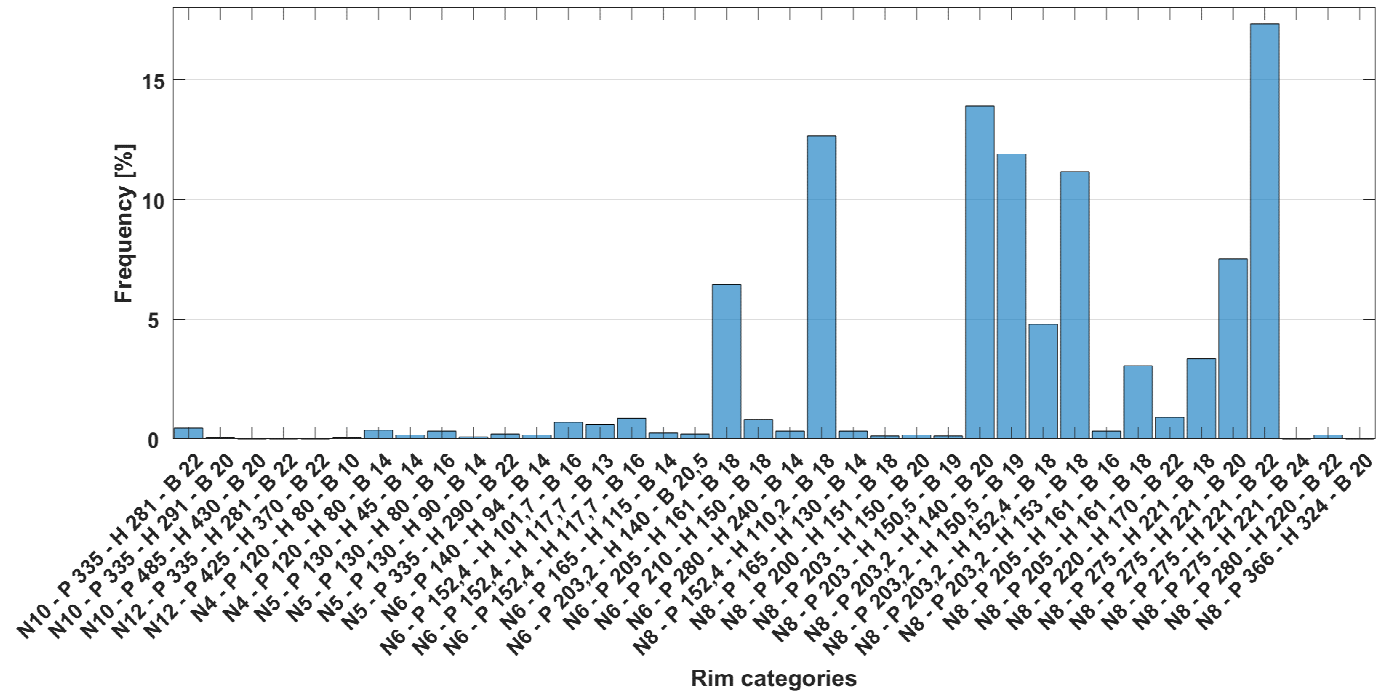

Fig. 7. Rim categories (38) with their occurrence frequency

Beside the frequency graph of Fig. 7, it is useful to perform also a further analysis, i.e. a graphical clustering analysis. It can be particularly useful, because it allows evidencing which of the 38 categories (or, better, of the 10 most frequent categories) have characteristics similar enough to be grouped together, thus further reducing the categories which can be satisfied by a single adapter with suitable (universal) features. The clustering analysis makes a wide use of graphical tools, such as multi-dimensional scatter plots and grouping algorithms, e.g. the GDBSCAN [27]. Indeed, a cluster can be easily identified by a spatial thickening of marker/points within these plots (density-based cluster modelling [28]). At this regard, in Fig. 8 there is a 5-dimension graph of the coupling categories and the two clusters are evidenced; the identification of the two clusters was made both visually and with the "dbscan" function implemented in MATLAB ${ }^{\odot}$ (by The Mathworks, Natick, Massachusetts, USA). Three dimensions of the graph are defined by the position of the category marker in the virtual space (the pitch circle diameter on the x-axis, the central bore diameter on the $y$ axis, the number of bolts on the z-axis), other two dimensions are defined by the colour scale, representing the bolt diameter, and by the marker size, representing the frequency of use on tractors of that class of coupling (the greater the radius of the circles, the greater the occurrence of finding that combination of parameters on the market).

The red and black circles in Fig. 8 individuate two clusters, i.e. two sets of categories that have characteristics suitable to be matched each by a single adapter. The two clusters were selected both visually and by means of the MATLAB ${ }^{\odot}$ function dbscan. Indeed, the elements of cluster " 1 " (N8P275-H221-B18, N8-P275-H221-B20, N8-P275-H221-B22) differ only in the diameter of the bolts (spanning from 18 to $22 \mathrm{~mm}$ ), while the elements of cluster "2" (N8-P203.2-H140-B20, N8-P203.2H150.5-B19, N8-P203.2-H152.4-B18, N8-P203.2-H153-B18) differ in the centring diameter (ranging from 140 to $153 \mathrm{~mm}$ ) and in the diameter of the bolts (spanning from 18 to $20 \mathrm{~mm}$ ).

An adapter suitable for categories of cluster 1 can have the bolt holes wide enough to fit the bolts of the larger category and, consequently, the holes will be suitable also for all smaller bolt diameters.

Instead, an adapter suitable for categories of cluster 2, will be equipped with a set of alignment rings to be fitted around the centring cylinder of the hub. In this way, it is possible to group together all the coupling categories that have an alignment hub diameter between 140 and $153 \mathrm{~mm}$. Moreover, if the alignment hole is further enlarged and the holes for the bolts are shaped like radial slots (overall dimensions: width $21 \mathrm{~mm}$, length $23 \mathrm{~mm}$ ), it will be possible to include two more categories within 
this cluster (N8-P205-H161-B16, N8-P205-H161-B18), i.e. the one with the bolt diameter of $16 \mathrm{~mm}$ and the one with the bolt diameter of $18 \mathrm{~mm}$. This modification should be taken into account in the development of the flange, especially, if considering that the category N8-P205-H161-B18 is increasingly being used in the most recent models of tractors.

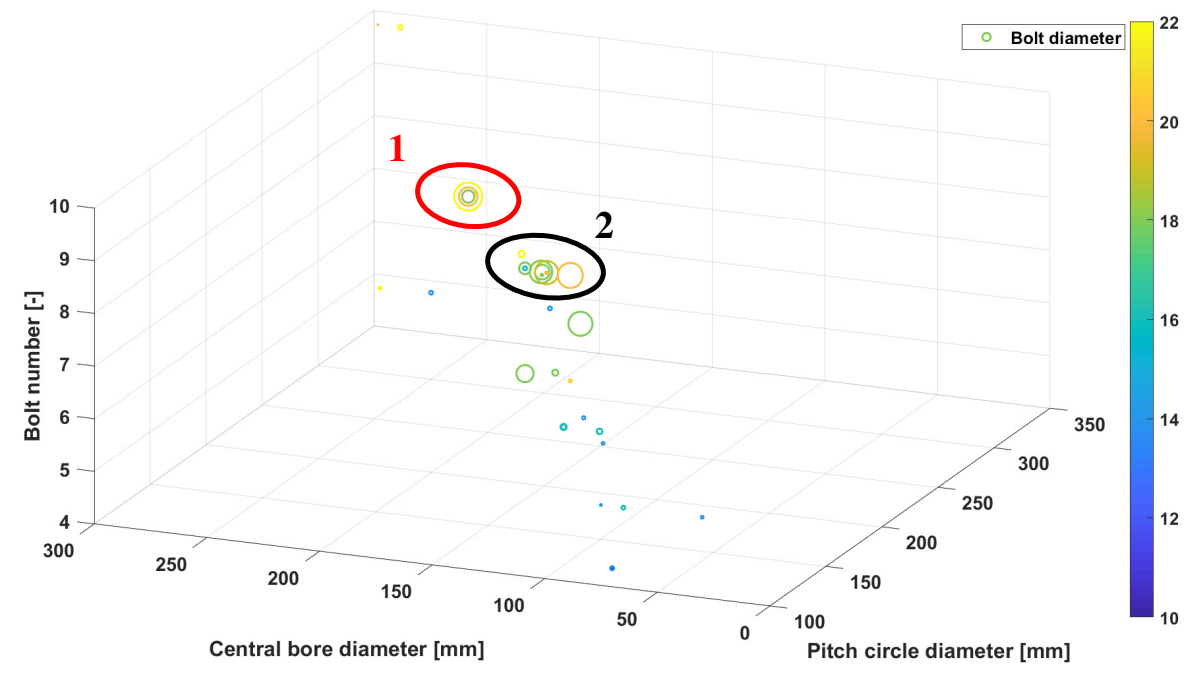

Fig. 8. Clustering analysis of rim categories; the two evidenced thickenings of markers indicate two clusters to be considered for development of the adapter

Finally, an adapter built on the basis of cluster 1 features will manage to fit to 678 models, whilst an adapter built on the basis of cluster 2 features will fit 1084 models, corresponding respectively to $28 \%$ and $45 \%$ of the reduced dataset. The results of the statistical analysis can be appreciated by observing the CAD drawings of the adapters, which have been designed based on these evidences. For example, in Fig. 9 is reported the adapter based on cluster 2. In this case, a single adapter (transparent in the figure, having the outer diameter of $520 \mathrm{~mm}$ ) can be used to connect the 6 illustrated different hub fixing systems (in darker grey) to the test rig. Adapters from A to D have also an alignment ring inside the central bore, as this is greater than the centring diameter on the hub (the bore diameter is $161 \mathrm{~mm}$ to fit the categories shown in $\mathrm{E}$ and $\mathrm{F}$ ).

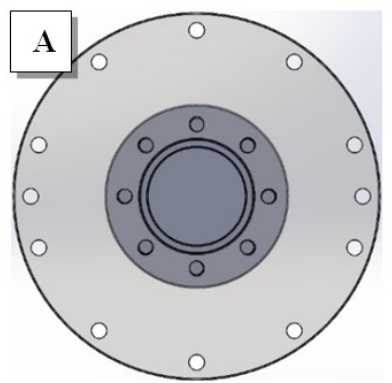

N8-P203.2-H140-B20

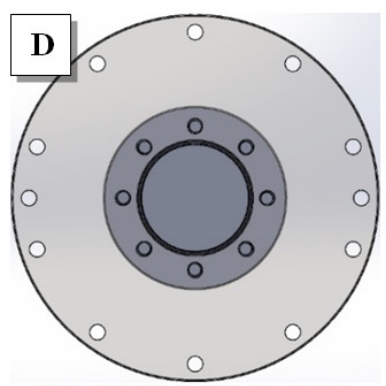

N8-P203.2-H153-B18

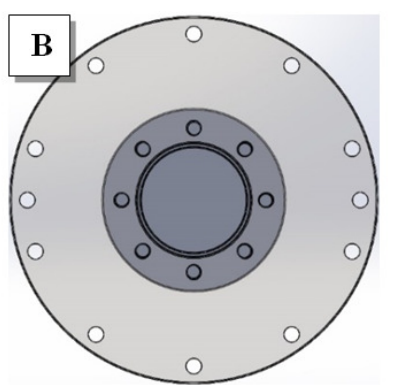

N8-P203.2-H150.5-B19

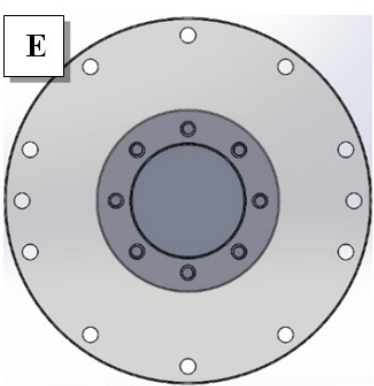

N8-P205-H161-B16

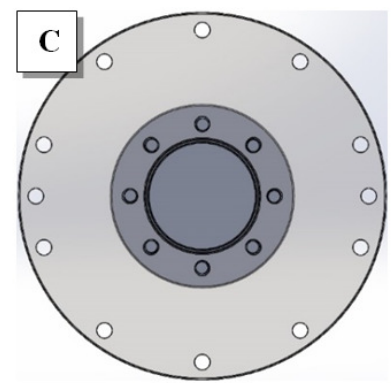

N8-P203.2-H152.4-B18

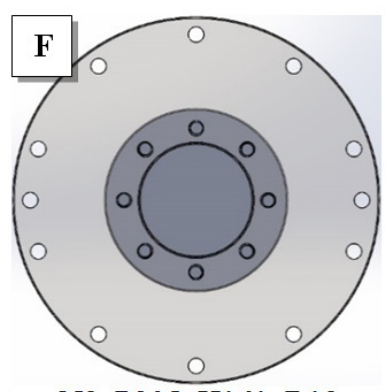

N8-P205-H161-B18

Fig. 9. Hub adapter designed to fit the 6 categories of the axle shaft fixing system grouped in cluster 2 (each category is indicated below the related sub-figure)

Subsequently, a more precise setting of the parameters used in the dbscan function allowed adding another important category to cluster 2. This category is the one defined as N6-P205-H161- 
B18 and it was initially discarded as it had 6 bolts instead of 8 . However, by properly positioning the holes (Fig. 10), it is possible to include also this category in the same adapter. Thanks to this improved layout, this adapter is able to be connected up to 1283 tractor models, corresponding to $53 \%$ of the models below $100 \mathrm{~kW}$. The two adapters are now able to test $81 \%$ of all the investigated models.
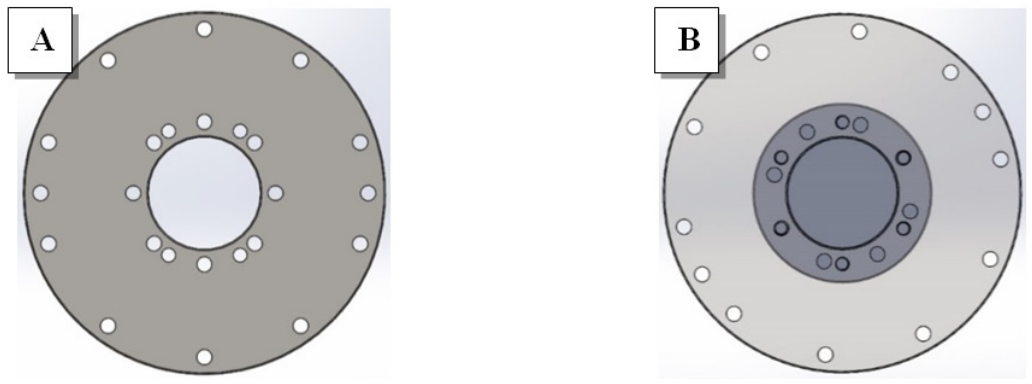

Fig. 10. Hub adapter modified after redefining cluster 2: A - new geometry; B - adapter mounted on a N6-P205-H161-B18 axle shaft category with 6 bolts

\section{Conclusions}

A systematic study of the different rim types has been performed in three phases. The aim was to develop, if possible, a universal component to be connected to the tractor axle shafts to let a test rig detect the tractor performances (torque and power) at the wheels. The starting point was an analysis of the international standards and market tractors. Although the ISO 5711 standard proposes 8 categories of wheel-to-hub couplings, characterized by many dimensional parameters, the study exposed here has demonstrated that this standard is followed by only $43 \%$ of the tractors on the market and that only four parameters are interesting for the development of the hub adapter. Thanks to a statistical analysis, it was possible to identify which categories of wheel-to-hub couplings are most used by manufacturers. Then, a cluster analysis allowed grouping some rim categories together in two sets or clusters. The combination of the design features of the rim categories belonging to these two clusters guided us in the design of 2 adapters, in this case matching up to $81 \%$ of the models. Therefore, by renouncing to have a total coverage of the tractor models, not worthy if considering the real distribution of models, but focussing only on the most frequent categories of couplings, the whole project of the experimental equipment has been optimized and important saving of money will be scored (only two pairs of adapters will be built, thus maximizing the cost-benefit ratio).

\section{Acknowledgements}

This research was developed within the "BIO-TRACT-EFFICIENCY" ("Experimental investigation on the efficiency of agricultural machines powered with different fuels") internal research project, grant number TN200F. Moreover, the authors wish to thank the "Consorzio Agrario di Bolzano" (the "Farmers' cooperative of Bolzano", Italy; www.ca.bz.it/), which allowed the authors to perform some direct measurements on their tractors.

\section{References}

[1] Froment Dynamometers. Froment SIGMA 50 dynamometers 2018. [online][18.03.2020] Available at: http://www.fromentdynamometers.com/sigma-50-dynamometers.html.

[2] Bietresato M., Malavasi M., Mazzetto F. Set-up of integrated system for real-time detection and recording of many engine parameters of agricultural machines during dyno tests. Proc. 18th Int. Sci. Conf. Eng. Rural Dev. ERD 2019, Jelgava (Latvia): 2019, pp. 160-173.

DOI: 10.22616/ERDev2019.18.N187.

[3] Bietresato M, Mazzetto F. Ideation, realization and experimentation of prototype device for measuring farm tractor fuel consumption during dyno tests. Eng. Rural Dev., vol. 17, 2018, pp. 362-372. DOI: 10.22616/ERDev2018.17.N446.

[4] Bietresato M, Caligiuri C, Bolla A, Renzi M, Mazzetto F. Proposal of a Predictive Mixed Experimental- Numerical Approach for Assessing the Performance of Farm Tractor Engines Fuelled with Diesel-Biodiesel-Bioethanol Blends. Energies 2019; 12:2287.

DOI: $10.3390 /$ en 12122287. 
[5] Bietresato M., Friso D. Durability test on an agricultural tractor engine fuelled with pure biodiesel (B100). Turkish J Agric For 2014, 38. DOI: 10.3906/tar-1302-51.

[6] Bietresato M., Caligiuri C., Renzi M., Mazzetto F. Use of diesel-biodiesel-bioethanol blends in farm tractors: first results obtained with a mixed experimental-numerical approach. Energy Procedia 2019; 158, pp. 965-971. DOI: 10.1016/j.egypro.2019.01.237.

[7] Pochi D., Fanigliulo R., Pagano M., Grilli R., Fedrizzi M., Fornaciari L. Dynamic-energetic balance of agricultural tractors: active systems for the measurement of the power requirements in static tests and under field conditions. J Agric Eng 2013, 44. DOI: 10.4081/jae.2013.s2.e84.

[8] Malcolm K. Green, Bill A. Stout, Stephen W. Searcy. Instrumentation Package for Monitoring Tractor Performance. Trans ASAE 1985; 28, 346-349. DOI: 10.13031/2013.32255.

[9] Van N.N., Matsuo T., Tastuya K., Shigeki I. Transducers for measuring dynamic axle load of farm tractor. Bull Fac Agric - Saga Univ 2009; 94, pp. 23-35.

[10]Feng L., Chen W., Wu T., Wang H., Dai D., Wang D., et al. An improved sensor system for wheel force detection with motion-force decoupling technique. Measurement 2018; 119, pp. 205217. DOI: 10.1016/j.measurement.2018.01.066.

[11] Weiblen W., Hofmann T. Evaluation of Different Designs of Wheel Force Transducers. SAE Tech Pap 1999-01-1037 1999. DOI: 10.4271/980262.

[12] Kistler Group. RoaDyn S6XT sp wheel force transducer for heavy commercial and special vehicles 2020. [online][18.03.2020] Available at: https://www.kistler.com/en/product/type9262a1/

[13]ASTA Technologies. Roller Test Bed 2020. [online][23.03.2020] Available at: http://astatechnologies.com/products/test-rigs-design-and-manufacturing/roller-test-bed.php

[14] Renk. All-wheel roller dynamometer 2020. [online][18.03.2020] Available at: https://www.renkag.com/en/products-and-service/products/test-systems/agricultural-vehicles/.

[15]Editor Agri-Mechanics. RTS Delivers Hub-Connected Ag-Machinery Test System 2017. [online][23.03.2020] Available at: http://www.agri-mechanics.com/rts-delivers-hub-connectedag-machinery-test-system/

[16] ISO-International-Standard-Organization. ISO 5711:1995(E) - Tractors and machinery for agriculture and forestry - Wheel-to-hub fixing dimensions. 1995.

[17]FEDERUNACOMA - Federazione Nazionale Costruttori Macchine per l'Agricoltura / Italian Agricultural Machinery Manufacturers Federation. Revisione macchine agricole e Formazione operatori - Audizione Commissione Agricoltura del Senato. Roma, Italy: 2015.

[18] Bietresato M., Calcante A., Mazzetto F. A neural network approach for indirectly estimating farm tractors engine performances. Fuel 2015, 143. DOI: 10.1016/j.fuel.2014.11.019.

[19] ISO-International-Standard-Organization. ISO 5711:1995(E). 1995.

[20] Intelligence C., Urbain J. What is Cluster Analysis? JayurbainCom n.d.

[21] Gentle J.E., Kaufman L., Rousseuw P.J. Finding Groups in Data: An Introduction to Cluster Analysis. vol. 47. 1991. DOI: 10.2307/2532178.

[22] Nadif M., Govaert G. Cluster Analysis. Data Anal., London, UK: ISTE; 2010, pp. 215-255. DOI: 10.1002/9780470611777.ch7.

[23] Needham M., Hodler A. Graph Algorithms Practical Examples in Apache Spark \& Neo4j. Oreilly \& Associates Inc; 2019.

[24]Dassault Systèmes. 3D Design \& Engineering Software - Dassault Systèmes 2020. [online][23.03.2020] Available at: https://www.3ds.com/

[25] CEMA aisbl - European Agricultural Machinery Industry Association. Stable year for tractor registrations in 2019 Total Registrations - 2019 2020. [online][18.03.2020] Available at: http://pr.euractiv.com/pr/stable-year-tractor-registrations-2019-202021.

[26] Bartolozzi F. Trattori europei sprofondo rosso. Macch e Mot Agric 2019, pp. 4-10.

[27] Sander J., Ester M., Kriegel H-P., Xu X. Density-Based Clustering in Spatial Databases: The Algorithm GDBSCAN and Its Applications. Data Min Knowl Discov 1998; 2, pp. 169-194. DOI: 10.1023/A:1009745219419.

[28] Kriegel H., Kröger P., Sander J., Zimek A. Density-based clustering. WIREs Data Min Knowl Discov 2011; 1, pp. 231-240. DOI: 10.1002/widm.30. 